\title{
Temperature Impact on Non-Linear Inductors in Operating Conditions for SMPS
}

\author{
Daniele Scirè, Student, IEEE, Marco Ventimiglia, Giuseppe Lullo, Member, IEEE, and Gianpaolo \\ Vitale, Senior Member, IEEE
}

\begin{abstract}
The exploitation of power inductors outside their linear region in switching converters requires a detailed description of the magnetization curve that is often not included in the datasheets; besides, the temperature of the inductor must be taken into account. This paper shows how to characterize the behavior of an inductor when it is operated up to saturation and its temperature rises. In order to characterize the inductor in real operating conditions, a dedicated measurement rig has been developed. It consists of a switching converter that includes the inductor under test and is controlled by a virtual instrument developed in LabVIEW. The characterization system was tested by retrieving the inductance and the magnetization curves vs. current for two commercial inductors at core temperatures up to $105^{\circ} \mathrm{C}$. The magnetic core is then characterized by the saturation current versus inductance, obtaining an expression for the whole family of inductor sharing the same core. Finally, we analyzed experimentally the thermal transient of the inductors in operating conditions confirming the fundamental role of temperature in changing the current profiles and the core saturation condition.
\end{abstract}

Index Terms - nonlinear magnetic, inductance measurement, power inductor, saturation magnetization, DC-DC power converter.

\section{INTRODUCTION}

$I^{2}$ NDUCTORS are crucial components of switch-mode power supply (SMPS) circuits where they are employed to store and release energy according to the operation of power switches. Currently, the inductor is often the most cumbersome component in an SMPS. In the next future, the continuous increase in switching frequencies will allow the integration of magnetic components with embedded windings directly in the PCB, a practical and cost-effective solution for a wide range of applications [1]. In order to increase the power density and reduce the weight of modern SMPS, the operation of inductors in such converters under moderate saturation is gaining interest. The behavior of inductors approaching saturation has already been of interest for low-frequency applications [2], [3] due to its influence on the current in the circuit where it is employed. Also, in higher frequency applications concerning SMPS, the inductor can be advantageously exploited outside of the linear zone to reduce its cost and weight [4]-[6].

D. Scirè., M. Ventimiglia and G. Lullo are with Dipartimento di Ingegneria, Università degli Studi di Palermo, 90128 Palermo, Italy (e-mail: daniele.scire@unipa.it, giuseppe.lullo@unipa.it).
Among available magnetic materials for the inductor core, ferrite exhibits low losses and relatively high saturation induction; for this reason, it reveals suitable for power electronics applications [7]. In SMPS the inductor is operated with a DC current offset and AC components imposed by the converter operation. Moreover, the inductor's non-linear operation modifies the triangular-shaped waveform of its current [8], raising its peak value and leading to a situation that can affect the stability of the converter. In fact, outside the linear zone, the current peak's growth raises the losses, increasing the temperature. In general, the rise of the temperature influences the inductance, lowering its value. It can bring to a runaway situation requiring a more detailed thermal analysis [9]-[11].

This paper focuses on the role of the inductor temperature when saturation is exploited. Here saturation is intended as the condition in which the differential inductance is reduced to half of its maximum value [4]. Recently, some papers analyzed SMPS inductors operated outside the linear zone. In [8], [12][16], the time-domain waveforms were derived and compared to the experimental ones, with a good agreement, in different steady-state conditions using different models and approaches. Issues related to modelling the temperature effects have been assessed in [5], where current profiles for different temperatures of the inductor are obtained in steady-state. In [17] the thermal transient was analyzed, showing that, despite a lowering of the value of inductance due to the increase of temperature, small power inductors operating under partial saturation are not subjected to thermal drift. A model to reproduce thermal transient in SMPS simulations showing different current waveforms during the thermal transient is proposed in [10]; this approach adopts a behavioral model based on electrical parameters where the temperature is obtained as output. It should be noted that [17] derives the inductor temperature starting from the inductor total power dissipation and the inductor thermal resistance; even if the former can be calculated, the latter may depend on non-predictable conditions such as circuit layout, air flow and dust due to ageing. Moreover, it considers a fixed frequency control (FFC) for the converter. In this paper, we will discuss how the converter can be preserved by a variable frequency control (VFC).

G. Vitale, is with Institute for High Performance Computing and Networking (ICAR), National Research Council (CNR), Palermo, Italy (e-mail: gianpaolo.vitale@icar.cnr.it). 
"This work has been submitted to the IEEE for possible publication. Copyright may be transferred without notice, after

which this version may no longer be accessible."

All the papers mentioned above agree to adopt basic low power DC/DC converters (buck or boost) as case studies to test their methods since results can be re-assessed for few $\mathrm{kW}$ output power DC/DC converters. However, we show that, as the power is augmented, increased losses will contribute to lower the output voltage requiring a feedback correction. The voltage feedback can lead to overtake the current limit. Indeed, we aim to show the inductor operation in two different situations starting from the temperature measured on the inductor. Even if it requires an additional measurement, i.e., the inductor temperature, it allows monitoring the proper and safe operation (sustainable saturation operation) in real operating conditions. Differently from the linear operation, the temperature of the inductor strongly influences the overall stresses. Besides, the effect of the feedback is discussed.

The inductor exploitation outside its linear zone requires a detailed characterization that often goes beyond the basic information supplied in the manufacturers' datasheets; in fact, saturation is mostly defined as the point in which the inductance is reduced by $10 \%$ only, and the value of such a current is given at environment temperature [10]. However, the temperature is subjected to a relevant increase especially exploiting saturation. Besides, it is influenced by external factors like PCB layout, the proximity of the power switch heatsink, and air-cooling that are difficult to take into account. For this reason, we propose to measure the temperature at a suitable point of the inductor, retrieving information based on this value. A dedicated measurement system has been set-up for this purpose by the authors.

Measurement systems to retrieve the inductance value of inductors with ferrite core have been proposed in [18] and recently in [19]. However, those two papers deal with inductors under low power sinusoidal solicitations; therefore, the solutions are of limited interest for power electronics applications where inductors are simultaneously interested by a DC offset (average output current) and an AC signal (current ripple). Since the AC signal has a high-frequency content and must be amplified to a suitable level before the acquisition, an $\mathrm{RF}$ amplifier is often needed. Even if a lock-in amplifier can improve the noise-to-signal ratio [20], this approach generally leads to an expensive system. A measurement system addressing real operating conditions has recently been proposed in [21], where a large signal characterization is performed by using two suitable AC and DC generators to supply the inductor. An automated measurement system for core loss characterization is proposed in [22], where the attention is focused on transformers and inductors for SMPS. The heat generation and thermal model issues are discussed in [23] and [24], considering the inductor operated in the linear zone. A commercial system has been developed for testing inductors in quasi-real operating conditions exploiting a DC-bias current generator and a triangular current ripple generator placed in parallel with the inductor under test [25]. In the literature, the proposed approaches try to supply the inductor under test by emulating the operating conditions. The same approach has been followed by [26] adopting an SMPS to improve the method of [27] based on a power amplifier for modeling core losses. It has to be underlined that all papers considering the temperature of the inductor,to characterize its behavior, do not analyze the distribution of the temperature inside the core.

Based on these considerations, this paper analyzes the results obtained with a switching converter, driven by an automated measurement system that performs the test of power inductors in real operating conditions. The feasibility of such a system was previously examined in [28], where the circuit of the SMPS was controlled by a LabVIEW virtual instrument in order to vary the load. In this paper, a new version of the circuit is presented, which exhibits the following improvements: a) the temperature is taken into account, which allows characterizing the inductor at constant temperature; b) the parasitic resistance of the winding is preliminarily estimated, and the related drop voltage is considered; c) the measurements are repeated to assess the mean value and the standard deviation of the results, giving an estimation of the error; d) a new inductor current sensor has been adopted to avoid any influence in the measurement; e) the PCB layout has been optimized to minimize parasitic inductances and consequent voltage ringing during commutation. The proposed system gives the core saturation curve versus inductance to be retrieved, as well as the magnetization curve profile, showing the inductance variation with the measured temperature. As an example of application, two commercial inductors have been characterized, including them inside the SMPS circuit, retrieving the inductance vs. current curves at different temperatures. Differently from [18], the proposed approach can be generalized for power inductors; it is cheaper compared to [22], which uses a temperature chamber, and a commercial system such as [25] and can be built for laboratory purposes. The inductor thermal behavior and modeling are relevant to the DC/DC converter design [23], [24]. Our contribution addresses this issue since the characterization always takes into account the temperature measured on the core. Finally, the knowledge on the inductor can be exploited in converters with different layouts since the measured temperature will represent the real operating conditions.

The paper is organized as follows. After the introduction, the main features of soft ferrites are presented in section II. Section III describes the DC/DC converter and the measurement system, while section IV contains a description of the virtual instrument developed to characterize the inductor and acquire the current profiles. Section V analyzes the error due to the method; two inductors model considering the temperature are presented in section VI, and finally, experimental results showing how the current profiles are influenced by temperature are given in section VII.

\section{Magnetic Materials FOR POWER EleCtronics}

Among different magnetic materials used in power electronics, ferrites are common due to their low losses in a 
"This work has been submitted to the IEEE for possible publication. Copyright may be transferred without notice, after

which this version may no longer be accessible."

wide frequency range and high specific resistivity. Besides, the magnetic properties are isotropic, the Curie temperature is around $700^{\circ} \mathrm{C}$, several fabrication techniques can be used and different shapes can be obtained. The saturation induction ranges from $0.25 \mathrm{~T}$ to $0.45 \mathrm{~T}$ (lower than iron and cobalt alloys) and the initial relative permeability ranges from 1,000 up to 20,000 for some new materials [7]. A higher cross-sectional area allows the use of ferrite for high power inductors to prevent saturation, increasing the weight and the cost of the component. The bulk resistivity is always high; it permits to distinguish the behavior of MnZn (0.1-10 $\Omega \cdot \mathrm{m})$ and NiZn $\left(10^{4}-10^{6} \Omega \cdot \mathrm{m}\right)$, depending on temperature and frequency. Such high resistivity is advantageous in keeping low the core losses even at higher frequencies where eddy current losses are dominant: the losses are inversely proportional to the resistivity of the core material and increase approximately with the square of the frequency. Moreover, the parasitic capacitance short-circuits the crystal boundaries at high frequencies, further decreasing resistivity [7]. It should be remarked that the ferrites mentioned above are "non-microwave ferrites", meaning that they can be used only up to some hundreds of $\mathrm{MHz}$. The magnetization curve of ferrites depends both on physical properties and on the manufacturing process. For this reason, the B-H loops at several frequencies and temperatures need to be measured directly on samples. For ferromagnetic materials, it is convenient to adopt the differential inductance, $L_{d i f}$, defined as the incremental ratio on the plane $\Psi$ vs. $i$ (Fig. 1), where $\Psi$ is the total flux linkage, $i$ is the magnetizing current, $\Phi$ is the flux linkage per turn and $N$ is the number of turns [4].

$$
L_{\text {dif }}=\frac{d \Psi}{d i}=N \frac{d \Phi}{d i}
$$

The practical limit in power electronics (the so-called linearity limit or saturation point) is defined as the point where the differential inductance is reduced, due to saturation, to half of its maximum value [7]. This definition sometimes differs from the value adopted by manufacturers, who consider the point at which the inductance is reduced by $10 \%$ only. It can be noted that the differential inductance coincides with the general inductance definition $(L=\Psi / i)$ inside the unsaturated region. When the flux density exceeds saturation, due to reduction of inductance, a higher current variation is needed to reach the same flux variation as in the linear region. The effect of heating can be appreciated by comparing the hysteresis curves taken at different temperatures. In general, heating flattens the hysteresis curve, lowering the value at which saturation occurs [29]; such a lowering, due to the higher temperature, corresponds to a decrease of permeability and, consequently, to a lower inductance value. The abrupt fall of the inductance, caused by a similar variation of the permeability, can be avoided by introducing small air-gaps inside the magnetic path; it results in the "shearing" of the hysteresis loop [29]. Consequently, the permeability remains constant in a wide range, making the coil characteristics less dependent upon the initial permeability of the core. On the other hand, the lower inductance needs more turns with consequent more copper losses; thus, a trade-off is necessary [29]. The use of soft

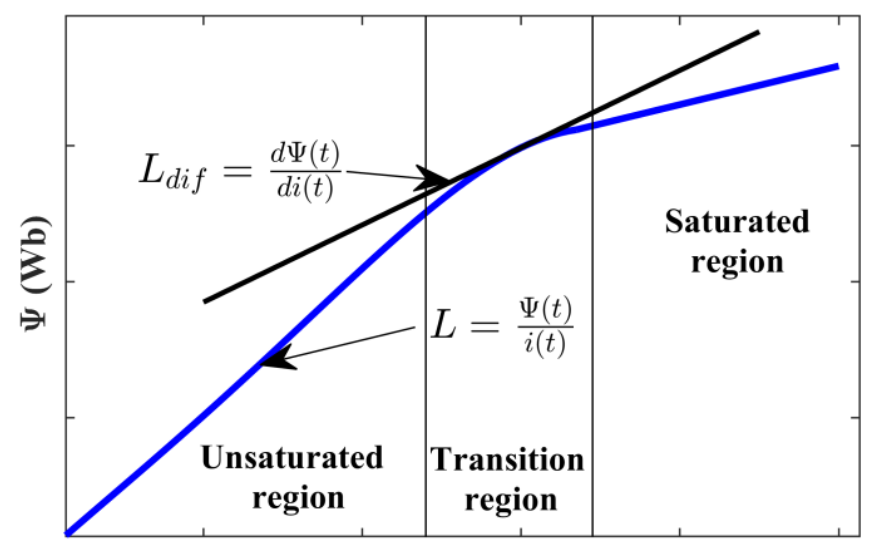

Current (A)

Fig. 1. Example of a magnetization curve for a ferrite core

saturation ferrites is an alternative to materials with air-gap core.

\section{THE DC/DC CONVERTER AND THE MEASUREMENT SYSTEM}

The circuit in which the inductors are tested is a buck converter working in continuous conduction mode (CCM). It is realized on a dedicated PCB. The converter switch is a power MOSFET IRFP150 with an IR2101 driver. The freewheeling diode is a MURB820PBF. The current through the inductor is measured by a Hall-effect current sensor ACS730 by Allegro.

The variable load is devised to obtain a constant current on a resistive load. A reference voltage, given by the output of a Digital to Analog Converter (DAC) MCP4822, controls an LM741 OP-AMP, which, together with an IRFP150 MOSFET and three $1 \Omega$ power resistors in parallel, connected between the source and ground, implement a current sink circuit. The MOSFET drain is connected to the buck converter's output, thus acting as a constant current resistive load. Due to power dissipation, the MOSFET is mounted on a suitable heatsink.

The Arduino Uno board, connected to a notebook via USB, varies the DAC output voltage, the frequency and duty cycle of PWM signal, according to the data received from LabVIEW; moreover, it acquires the temperature of the inductor by an LM35DZ sensor stuck onto the core through a thermal pad. This information is also useful to keep the inductor temperature constant during measurements. It should be noted that the temperature of the inductor on the surface of the core can be considered uniform for the geometry, size and type of the inductors under study [9].

The test rig comprises a DC linear power supply $(12 \mathrm{~V}, 10 \mathrm{~A})$, a digital oscilloscope Siglent SDS 1104X-E, a personal computer and the $\mathrm{PCB}$ with the $\mathrm{DC} / \mathrm{DC}$ converter including the IUT. For the measurements shown in this paper, the following parameters have been set: duty cycle of the DC/DC converter fixed at $50 \%$, switching frequency set automatically by the system to limit the current ripple of the inductor below $0.4 \mathrm{~A}$ (more details will be given in the next paragraphs), warm-up current equal to the saturation current that, at the lower 
"This work has been submitted to the IEEE for possible publication. Copyright may be transferred without notice, after which this version may no longer be accessible."
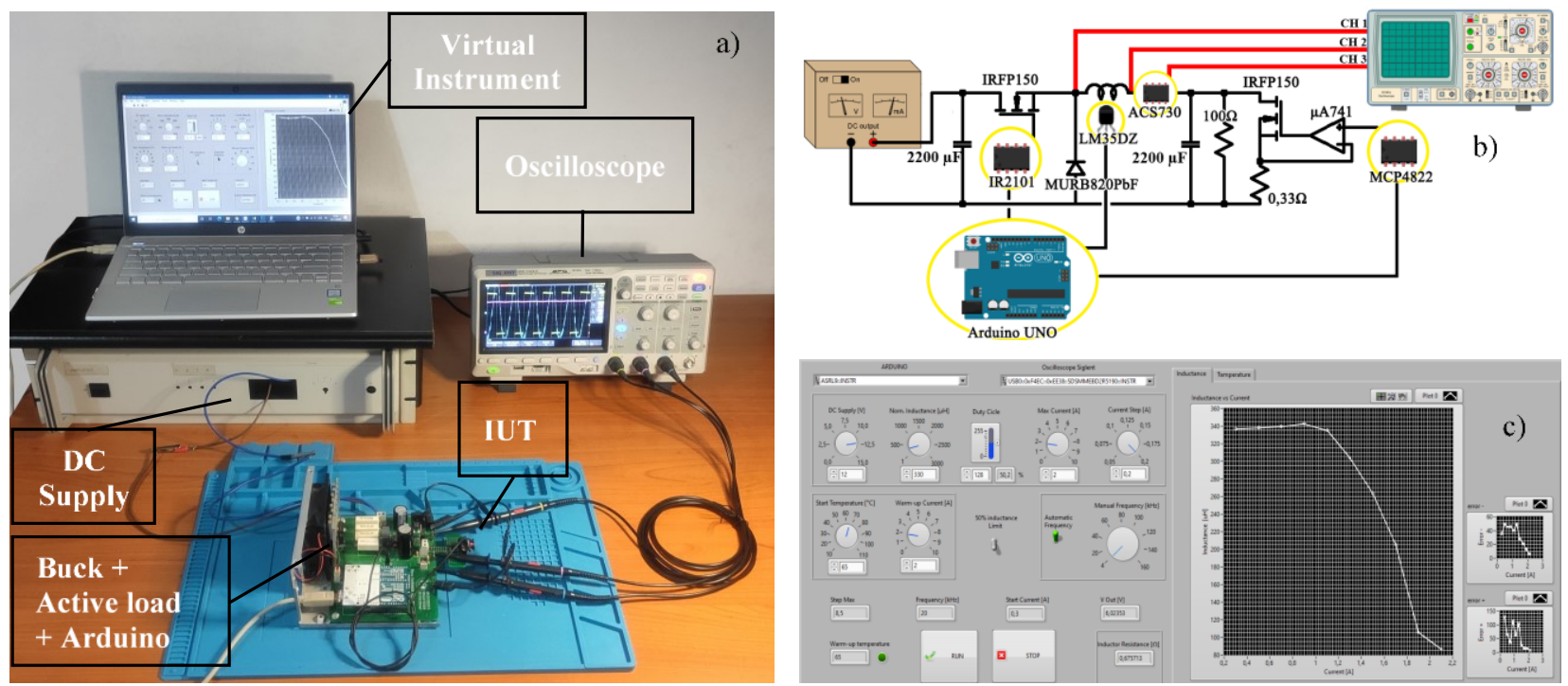

Fig. 2. a) Measurement setup. b) Simplified circuit schematic with the black components assembled on a PCB. c) Virtual instrument interface.

temperature step, gives the $50 \%$ of the rated inductance value. Figure 2a shows all the parts described before: the circuit PCB, the control PC, the benchtop power supply and the oscilloscope with its three probes to measure the current and the voltage across the inductor.

As already anticipated, the measurement system is based on a DC/DC buck converter, where the inductor under test (IUT) is a part of the circuit. The variable load allows varying the DC component of the inductor current during measurements. The tests are performed on the same inductor with different DC operating point to obtain the inductance $L_{d i f}$ versus the inductor current $i$ at different temperature points. In this way, the inductor is tested in real operating conditions.

The PWM square wave, controlling the switching MOSFET of the DC/DC converter, is generated by the Arduino Uno board with programmable frequency and duty cycle. Changing these parameters makes it possible to vary the amplitude of the current ripple in the IUT around the DC offset. The current signal, the input and output voltages of the DC/DC converter are acquired by the oscilloscope that sends data to the virtual instrument developed with National Instruments LabVIEW. Once the signals have been acquired, the following formula derives the differential inductance value, where $i$ and $V_{L}$ are the current through the inductor and its voltage, respectively.

$$
L_{\text {dif }}=\frac{V_{L}}{d i / d t}
$$

The current time derivative $(d i / d t)$ is calculated during the conduction time $\left(T_{\text {on }}\right)$ when the inductor is subjected to a constant voltage and the current grows like a ramp. Only about $90 \%$ of the ramp is considered for calculating the current slope, allowing a good estimation and excluding the oscillations during commutation; then, the current is sampled and its slope is retrieved based on linear regression. The current is sampled at $500 \mathrm{Ms} / \mathrm{s}$. The electric schematic of the buck converter is shown in Figure 2b.

\section{THE VIRTUAL INSTRUMENT}

The virtual instrument allows the management of the converter and the measurement system; besides, it arranges acquired data to be presented in graphic form. The main panel is shown in Fig. 2c. The instrument manages the converter parameters and acquires the measurements from the oscilloscope and the temperature sensor. Moreover, it can: (i) calculate the parasitic resistance of inductor; (ii) plot the value of inductance $L$ versus the inductor current $i$ for given temperature; (iii) visualize the curves of inductance; (iv) store all the measurements data on the computer memory for off-line post-elaboration. Several virtual potentiometers and switches are available, through which the user can set the following parameters before starting the measurement: a) the nominal value of inductance; b) the maximum DC current to reach; c) the duty cycle of the converter; d) the voltage of the external power supply; e) the temperature at which the measurement must be performed; f) the current to heat the inductor when necessary. Besides that, it is possible to set the switching frequency of the DC/DC converter manually. Alternatively, it can be automatically chosen by the program according to the pre-set parameters.

After the communications with the oscilloscope and the Arduino board are initialized, the measure can start. Firstly, the parasitic resistance of the inductor is calculated; then, the temperature is brought to the set value by powering the $\mathrm{DC} / \mathrm{DC}$ converter until the desired temperature is reached. Based on the geometrical characteristics of the core and on the material, its temperature can be assumed constant; for this reason, a thermochamber is not required [9]. The inductance evaluation is repeated for increasing DC current until the maximum preset current is reached; for each measurement, the core temperature is checked and eventually corrected by running or stopping the converter. An example of data retrieved by the virtual instrument is proposed in Fig. 3. The measurement process is schematized concisely in Algorithm 1. 
"This work has been submitted to the IEEE for possible publication. Copyright may be transferred without notice, after which this version may no longer be accessible."

\begin{tabular}{|c|c|}
\hline \multicolumn{2}{|c|}{ ALGORITHM 1} \\
\hline \multicolumn{2}{|l|}{ repeat } \\
\hline if Inductor_Temp $<$ Set_Temp & //Temperature Check \\
\hline Converter $\leftarrow \mathrm{ON}$ & //Turn on the converter \\
\hline \multicolumn{2}{|c|}{$\begin{array}{l}\text { Output_Load } \leftarrow \text { Warm-up_Load //Set a current to warm-up the inductor } \\
\text { end if }\end{array}$} \\
\hline if Inductor_Temp $>$ Set_Temp & //Temperature Check \\
\hline $\begin{array}{l}\text { Converter } \leftarrow \text { OFF } \quad \overline{/ / T u r n} \text { off the } \\
\text { end if }\end{array}$ & converter to cool down the inductor \\
\hline if Inductor_Temp $==$ Set_Temp & //Temperature Check \\
\hline Converter $\leftarrow$ ON & //Turn on the converter \\
\hline Voltage Acquisition & //Acquire voltage waveform \\
\hline Current Acquisition & //Acquire current waveform \\
\hline Inductance Calculation & //Compute Ldif via (2) \\
\hline $\begin{array}{l}\text { Out_Load } \leftarrow \text { Out_Load-Step_Load } \\
\text { end if }\end{array}$ & //Reduce load and increase current \\
\hline until Inductor_Current $<$ MaxCurrent & $\begin{array}{l}\text { //When the maximum current is } \\
/ / \text { reached, measurements stop }\end{array}$ \\
\hline Converter $\leftarrow \mathrm{OFF}$ & $/ /$ Turn off the converter \\
\hline
\end{tabular}

\section{ERROR ANALYSIS}

According to (2), inductance is calculated by dividing the voltage applied to the inductor (constant during $T_{o n}$ ) by the current slope. For the inductance calculation, the measurement of the current and its time derivative is repeated several times; the mean value of the calculated inductance is assumed as the true value, whereas the standard deviation of the results gives the error. Twelve acquisitions revealed, most of the time, as an acceptable trade-off, allowing the error to settle without overheating the inductor or excessively increasing acquisition time. By applying the error propagation on (2) and considering the voltage $V_{L}$ as not affected by any error, the following expression is obtained:

$$
\Delta L_{\text {dif }}=\frac{\partial}{\partial(D i)}\left(\frac{V_{L}}{D i}\right) \Delta(D i)=-\frac{V_{L}}{(D i)^{2}} \Delta(D i)
$$

where for the sake of clarity $D i$ indicates the time derivative of the current in the denominator of (2). It can be noted that the error $\Delta L_{\text {dif }}$ on the value of $L_{\text {dif }}$ decreases when the derivative value increases. It means that the inductance measurement improves outside the linear region, where the current slope increases.

\section{RESULTS ON INDUCTORS CHARACTERIZATION}

Two commercial inductors have been tested inside the developed buck converter; for each of them, the following information is retrieved: a) inductance versus temperature, b) saturation current versus temperature, c) magnetization curve. Then, the characterization of the core is compared with the data given by the manufacturer.

\section{A. Inductor 1}

The inductor 1 under test is the Coilcraft DO5010H-104 [30]. For this inductor, the datasheet shows an inductance of $100 \mu \mathrm{H}$ $\pm 20 \%$ with a saturation current, giving $10 \%$ inductance drop, of $3.0 \mathrm{~A}$. The inductance curve measured at $25^{\circ} \mathrm{C}$ is shown in Fig. $3 \mathrm{a}$, where the red circles are the mean inductance value for each current and the error bands represent the 95\% confidence interval. It can be noted that the value of inductance in the linear region lies within the tolerance interval given by the a)

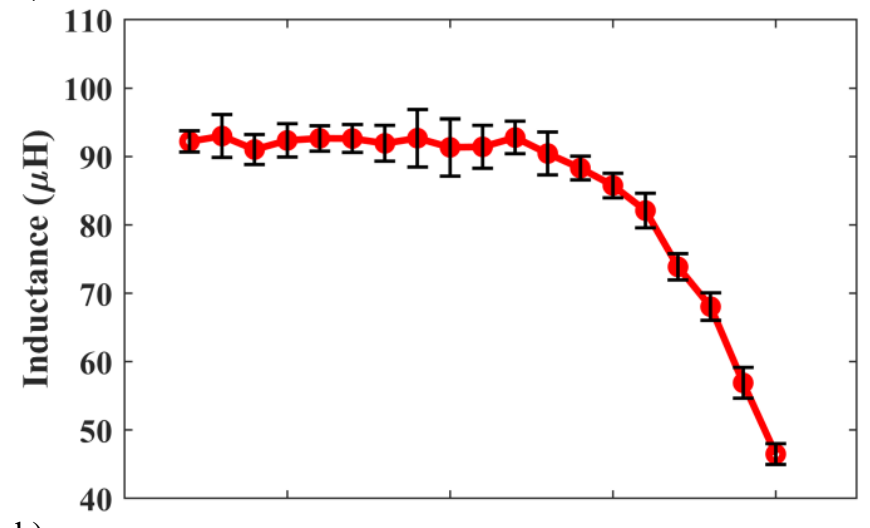

b)

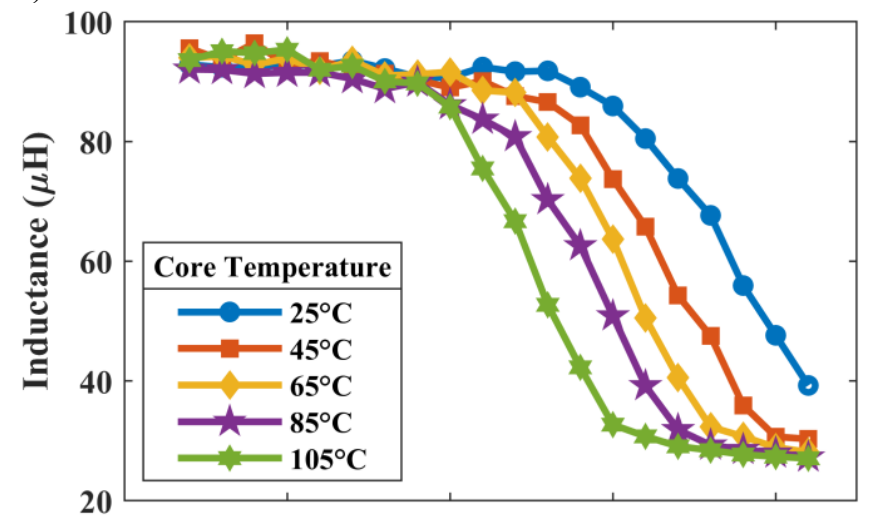

c)

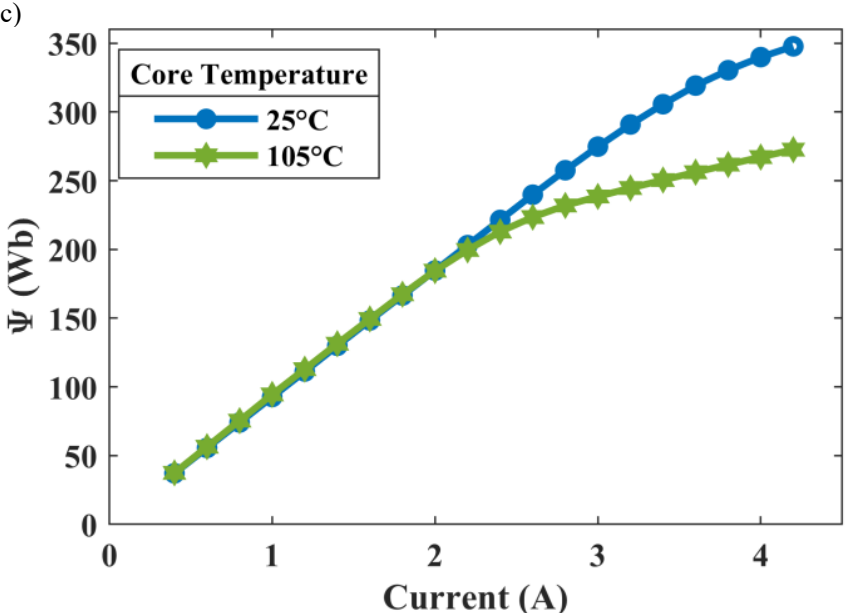

Fig. 3. Inductor 1 characteristics as a function of current and temperature: a) inductance vs. current at $T_{\text {core }}=25{ }^{\circ} \mathrm{C}$ with error band representing the $95 \%$ confidence interval; b) inductance vs. current, c) flux curve vs. current.

manufacturer. The manufacturer defines the saturation current as "the DC current at which the inductance drops 10\% from its value without current". From this definition and considering a measured mean value of the nominal inductance at $25{ }^{\circ} \mathrm{C}$ of 90 $\mu \mathrm{H}$, a value of about $3.2 \mathrm{~A}$ (at $81 \mu \mathrm{H}$ ) has been derived from the graph. Nevertheless, measurements were performed exceeding the $50 \%$ inductance drop according to the literature [7] and coherently with the exploitation range for power applications. The corresponding current has been found as 4.0 A at $25{ }^{\circ} \mathrm{C}$. In all measurements, the error remained inside the tolerance exhibited by the manufacturer and, as expected, it reduces for higher currents.

These measurements have been repeated at temperature steps 
"This work has been submitted to the IEEE for possible publication. Copyright may be transferred without notice, after which this version may no longer be accessible."

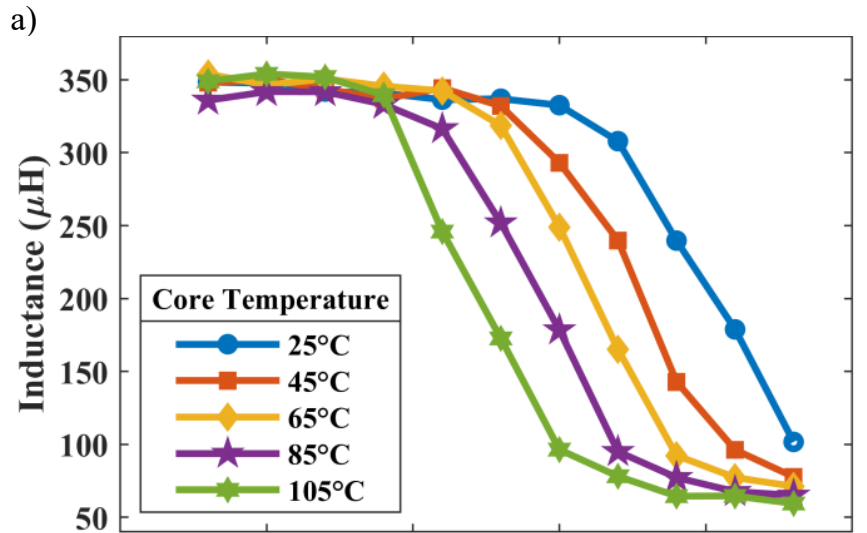

b)

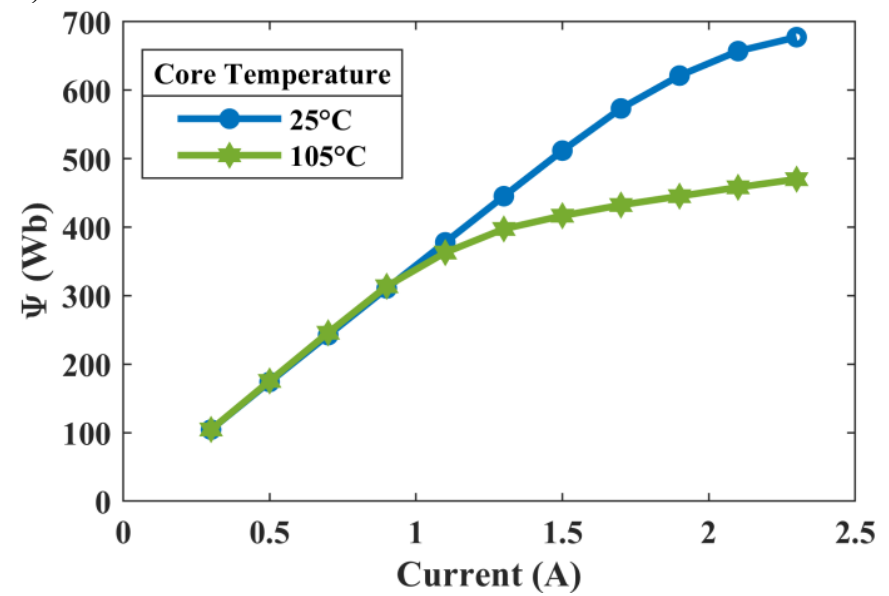

Fig. 4. Inductor 2 characteristics as a function of temperature: a) inductance vs. current, b) flux curve vs. current.

of $20^{\circ} \mathrm{C}$ up to $105^{\circ} \mathrm{C}$. The corresponding curves are reported in Fig. 3 b. It can be noted that the saturation current decreases as the temperature increases, meaning that the higher the temperature, the greater the variations in current with the same voltage applied. Besides, the slope of the inductance plot in the saturation region slightly increases with temperature. Based on the inductance curve, the magnetic flux versus current can be retrieved by:

$$
\Psi=\int L_{d i f} d i
$$

This curve has the same shape as the magnetization curve since the flux is proportional to the induction $B$ and the current to the magnetic field $H$. Hence, it can be used to verify the extension of the linear zone with temperature. The flux curve calculations at $25^{\circ} \mathrm{C}$ and $105^{\circ} \mathrm{C}$ are shown in Fig. 3c. These curves show that, at low temperatures, the linear zone has a broader range, while, for higher temperatures, the "knee" has reduced to about $2.3 \mathrm{~A}$. It represents the boundary of the linear zone.

\section{B. Inductor 2}

The inductor 2 under test is the Coilcraft DO5010H-334 [30]. This inductor belongs to the same series as the previous one and shares the same core. The datasheet gives an inductance of 330 $\mu \mathrm{H} \pm 20 \%$ and defines the saturation current based on a drop of $10 \%$ as $1.9 \mathrm{~A}$ at $25^{\circ} \mathrm{C}$. This value is confirmed experimentally in Fig. 4 a considering the plot at $25^{\circ} \mathrm{C}$, while the corresponding

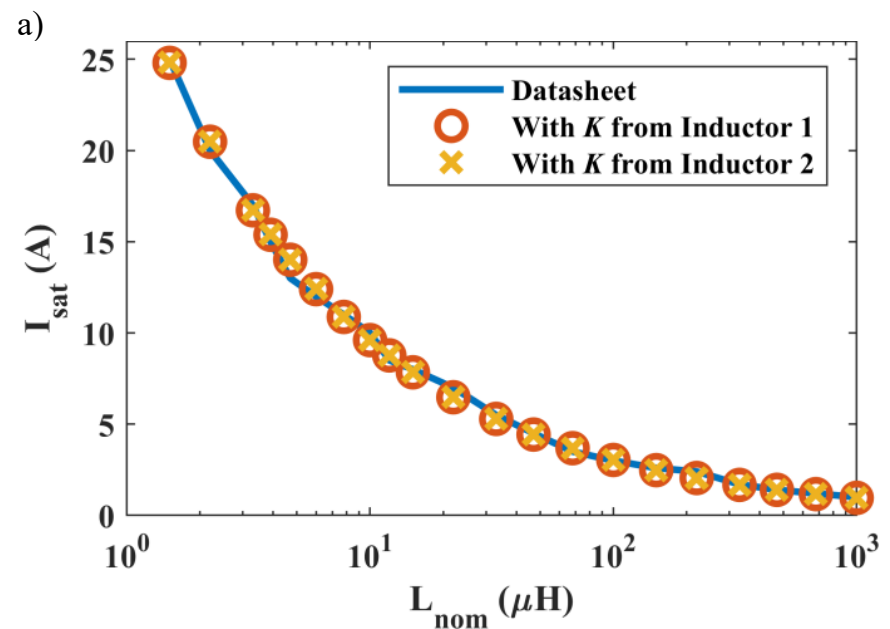

b)

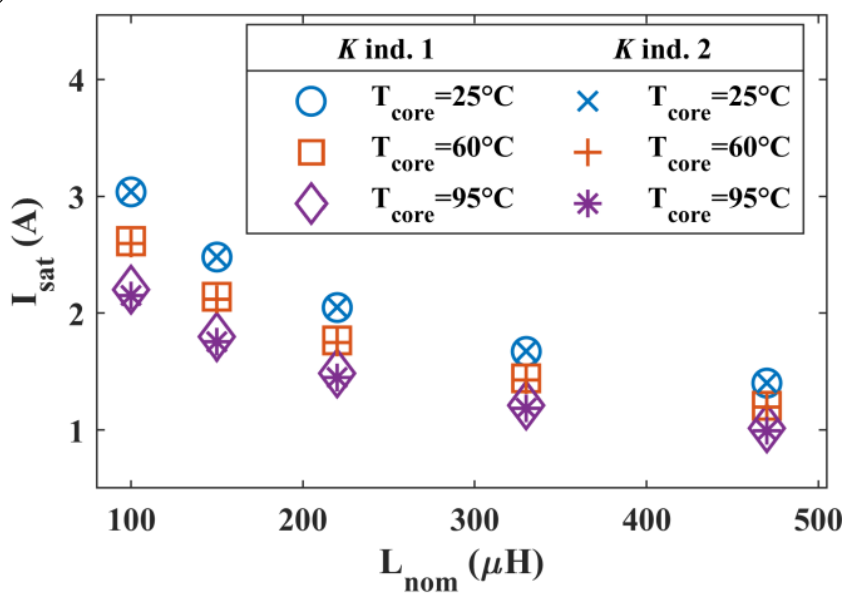

Fig. 5. Simulations of the saturation current vs. inductance characteristic of the inductor family. The $K$ factor of (7) has been extrapolated from the experimental data of inductor 1 and 2: a) Comparison of the simulation calculated with $K$ extrapolated from inductor 1 and inductor 2 with the information given by the datasheet at $T_{\text {core }}=25^{\circ} \mathrm{C}$. The abscissa is on a logarithmic scale for better visualization. b) Comparison of the simulated saturation currents obtained at different temperatures with $K$ extrapolated from inductor 1 and from inductor 2 .

flux curve is shown in Fig. 4b. The characterization is extended to $105{ }^{\circ} \mathrm{C}$ and to $2.2 \mathrm{~A}$ (current giving a drop of $50 \%$ of the rated value at $25^{\circ} \mathrm{C}$ ).

\section{Core characterization and comparison with data given by the manufacturer}

The two considered inductors exploit the same core. Based on the obtained results, the core can be characterized by the saturation current versus inductance, provided at different temperatures. The manufacturer delivers this information only at $25^{\circ} \mathrm{C}$. [30]. It should be remarked that the manufacturer defines the saturation current as "DC current at which the inductance drops $10 \%$ from its value without the current". It differs from the definition proposed by literature that considers a drop of $50 \%$; for the sake of comparison, the values based on obtained results are calculated adopting the manufacturer's definition.

The saturation current of an inductor $\left(I_{s a t}\right)$, as defined in [31], can be expressed as a function of the saturation flux density $\left(B_{\text {sat }}\right)$ with $l_{m}$ the mean magnetic path length, $N$ the number of turns and $\mu_{\text {sat }}$ the permeability of the core at saturation, which 
"This work has been submitted to the IEEE for possible publication. Copyright may be transferred without notice, after which this version may no longer be accessible."

is equal to the permeability of the core $\mu$ at zero current multiplied by a proportionality factor $C(0.5$ for the literature standard, 0.9 for the manufacturer's standard):

$$
I_{\text {sat }}=\frac{B_{\text {sat }} l_{m}}{\mu_{\text {sat }} N}=\frac{B_{\text {sat }} l_{m}}{C \mu N}
$$

The nominal inductance $L_{\text {nom }}$ is then calculated considering $A_{c}$, the area of the core section:

$$
L_{n o m}=\frac{\mu N^{2}}{l_{m} A_{c}}
$$

A new expression linking the saturation current to the nominal inductance can be obtained combining $(5,6)$ and through a proportionality factor $K$ containing the geometrical and physical properties of the core:

$$
I_{\text {sat }}\left(L_{\text {nom }}\right)=\frac{B_{\text {sat }}}{C} \sqrt{\frac{l_{m}}{\mu A_{c} L_{\text {nom }}}}=\frac{K}{\sqrt{L_{\text {nom }}}}
$$

From the experimental data regarding the inductor 1 at $25^{\circ} \mathrm{C}$, the proportionality factor $K$ can be evaluated by equation (7) multiplying both members by $\sqrt{L_{\text {nom }}}$, resulting in a value of $30.375 \times 10^{-3}\left(\mathrm{~A} \cdot \mathrm{H}^{0.5}\right)$. Using this value, the saturation current as function of the nominal inductance, can be retrieved. This method is valid for all the inductors of the same series, sharing the same ferrite core. Such a comparison is highlighted in Fig. 5a. showing an excellent fit with the datasheet information at $25^{\circ} \mathrm{C}$; the same result is obtained with the data retrieved from inductor 2, confirming the correctness of this approach.

Furthermore, the saturation current for inductor 1 ( $\left.I_{\text {sat-indl } 1}\right)$ depends from the temperature $\left(T_{\text {core }}\right.$ considered in $\left.{ }^{\circ} \mathrm{C}\right)$ and it can be interpolated by a straight line obtaining (8):

$$
I_{\text {sat-ind }_{1}}\left(T_{\text {core }}\right)=-0.0126 T_{\text {core }}+3.5168
$$

By multiplying (8) by the square root of the nominal inductance (a mean measured value of $90 \mu \mathrm{H}$ for the inductor 1 ), it is possible to retrieve the expression of the proportionality factor $K$ as a function of temperature; it should be noted that $K$ depends on the geometrical and physical properties of the core and is also temperature-dependent.

$$
K\left(T_{\text {core }}\right)=\left(-0.1195 T_{\text {core }}+33.363\right) \times 10^{-3}
$$

Figure $5 \mathrm{~b}$ shows the comparison between the data obtained from (7) with the expression of $K\left(T_{\text {core }}\right)$ extrapolated from the measurement of inductors 1 and 2 . Both expressions of $K$ result in similar values of the saturation current for the whole $L_{n o m}$ range; nonetheless, a smaller range of inductance is shown in Fig $5 \mathrm{~b}$ for the sake of tidiness. The quantitative data shown in Fig. $5 b$ are listed in Table I.

Since the expression of $K$ as the function of the temperature is known, it is possible to extend the manufacturer's information, including the temperature behavior, to the whole family of inductors sharing the same core. Such dependence is presented in a 3D surface in Fig. 6a, whereas the projection for a given nominal inductance (defined at $25^{\circ} \mathrm{C}$ ) is presented in Fig. $6 \mathrm{~b}$ and at constant core temperature in Fig. 6c. As

\begin{tabular}{|c|c|c|c|}
\hline \multicolumn{4}{|c|}{$K_{\text {ind }_{1}}\left(T_{\text {core }}\right)=\left(-0.1195 T_{\text {core }}+33.363\right) \times 10^{-3}$} \\
\hline$L_{\text {nom }}(\mu \mathrm{H})$ & $\begin{array}{l}\text { Sat. Current } \\
\text { (a) } 25{ }^{\circ} \mathrm{C}\end{array}$ & $\begin{array}{c}\text { Sat. Current } \\
\text { (a) } 60{ }^{\circ} \mathrm{C}\end{array}$ & $\begin{array}{c}\text { Sat. Current } \\
a, 95^{\circ} \mathrm{C}\end{array}$ \\
\hline 100 & 3.038 & 2.619 & 2.201 \\
\hline 150 & 2.480 & 2.139 & 1.797 \\
\hline 220 & 2.048 & 1.766 & 1.484 \\
\hline 330 & 1.672 & 1.442 & 1.212 \\
\hline 470 & 1.401 & 1.208 & 1.015 \\
\hline \multicolumn{4}{|c|}{$K_{\text {ind }_{2}}\left(T_{\text {core }}\right)=\left(-0.1271 T_{\text {core }}+33.585\right) \times 10^{-3}$} \\
\hline$L_{\text {nom }}(\mu \mathrm{H})$ & $\begin{array}{l}\text { Sat. Current } \\
\text { a } 25^{\circ} \mathrm{C}\end{array}$ & $\begin{array}{c}\text { Sat. Current } \\
\text { (a) } 60^{\circ} \mathrm{C}\end{array}$ & $\begin{array}{c}\text { Sat. Current } \\
\text { a } 95^{\circ} \mathrm{C}\end{array}$ \\
\hline 100 & 3.041 & 2.596 & 2.151 \\
\hline 150 & 2.483 & 2.120 & 1.756 \\
\hline 220 & 2.050 & 1.750 & 1.450 \\
\hline 330 & 1.674 & 1.429 & 1.184 \\
\hline 470 & 1.403 & 1.197 & 0.992 \\
\hline
\end{tabular}
previously remarked, $I_{\text {sat }}$ has a linear dependence from the
TABLE I

SATURATION CURRENTS AT DIFFERENT TEMPERATURES WITH K DERIVED FROM INDUCTOR $1(100 \mu \mathrm{H})$ OR FROM INDUCTOR $2(330 \mu \mathrm{H})$.

temperature (Fig. 6b). Moreover, at $105^{\circ} \mathrm{C}$, the saturation current is $36 \%$ lower than its corresponding value at $25^{\circ} \mathrm{C}$; this property is valid for all inductors sharing the same core. Such a drop cannot be ignored during the SMPS design process, especially when the inductor is exploited in partial saturation. Such analysis gives a complete characterization of the Coilcraft DO5010H-104 inductor family since each inductor of this series has the same ferrite core. This analysis can be extended to other inductors and is independent of the information available in the datasheet. It is worth noting that, although several inductors of the DO5010H-104 series cannot be measured by the system presented here due to the limited range of current provided by the power supply, however it is possible to extrapolate the characteristics of saturation current vs. core temperature through (7) for the whole family once that the proportionality factor $K$ is retrieved from the measurement of one suitable inductor of the same series.

Finally, this approach is helpful for retrieving information regarding the saturation and temperature behavior of power inductors in the non-linear region. The additional information can help the designers to adopt a cost-effective design of SMPS exploiting non-linear inductors, allowing them to address the adverse effects of saturation.

\section{EXPERIMENTAL ANALYSIS OF THE TEMPERATURE EFFECT}

In order to assess the temperature impact on the inductor behavior, the two inductors previously characterized have been further investigated in real operating conditions. Some current profiles are shown to highlight how the temperature of the inductor influences the current shape and, consequently, the performance of the converter. The tests have been conceived based on the models of the two inductors that have been previously retrieved. The knowledge of the model is crucial since it allows to forecast the maximum current imposed by the lowering of the inductance.

For each inductor the test was performed starting from environmental temperature, with a fixed duty cycle and load. The mean current through inductor 1 is equal to $3.2 \mathrm{~A}$, whereas the mean current through inductor 2 is equal to $1.7 \mathrm{~A}$. In both 
"This work has been submitted to the IEEE for possible publication. Copyright may be transferred without notice, after which this version may no longer be accessible."

a)

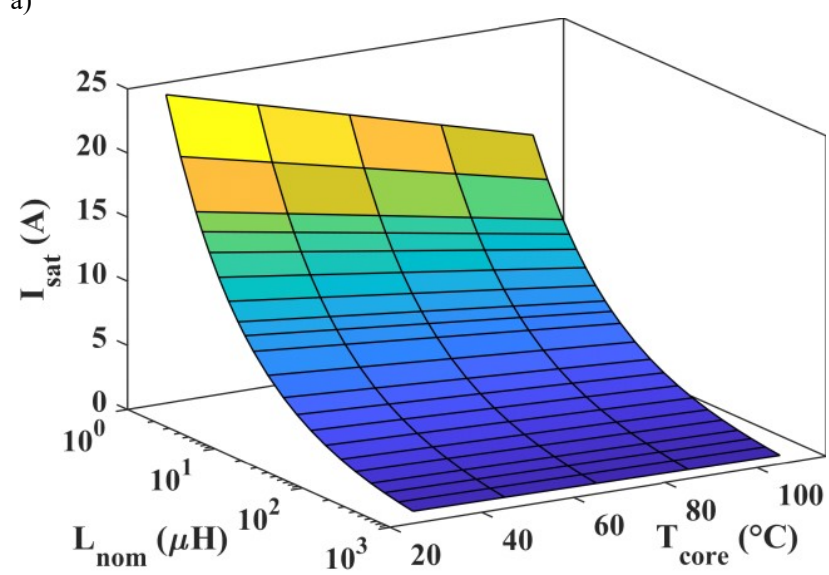

b)

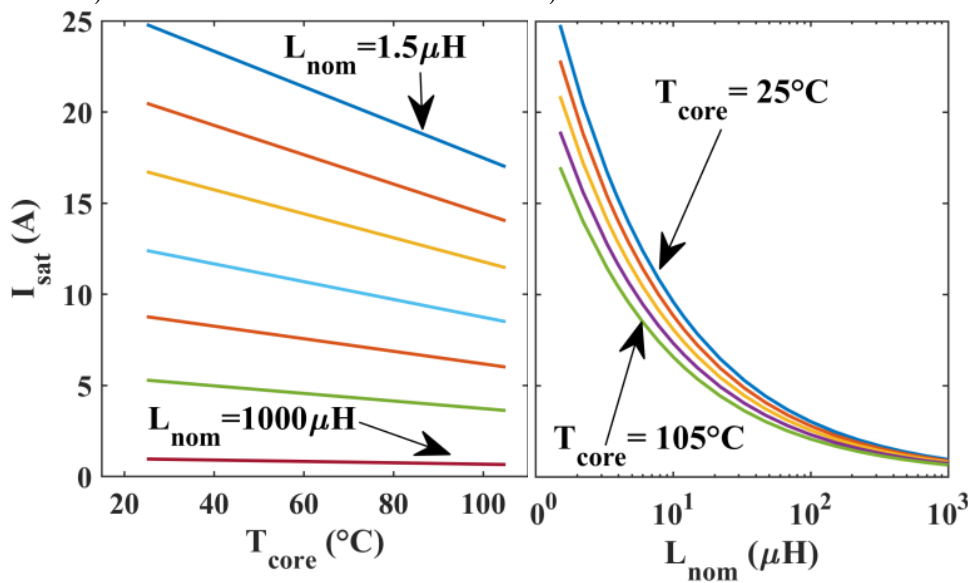

Fig. 6. Saturation current behavior for the inductor family simulated from (7) and (9): a) saturation current $\left(I_{\text {sat }}\right)$ vs. nominal inductance $\left(L_{\text {nom }}\right)$ vs. core temperature $\left(T_{\text {core }}\right)$, b) $I_{\text {sat }}$ vs $T_{\text {core }}$ for a given $L_{\text {nom }}$, c) $I_{\text {sat }}$ vs. $L_{\text {nom }}$ at constant $T_{\text {core }}$. The inductance axis is on a logarithmic scale for better visualization.

a)

b)

c)

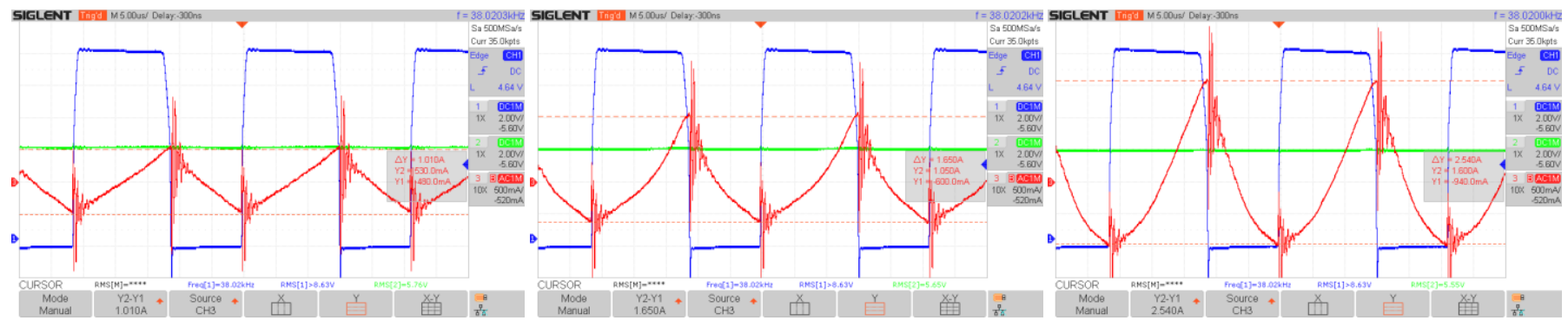

d)

e)

f)

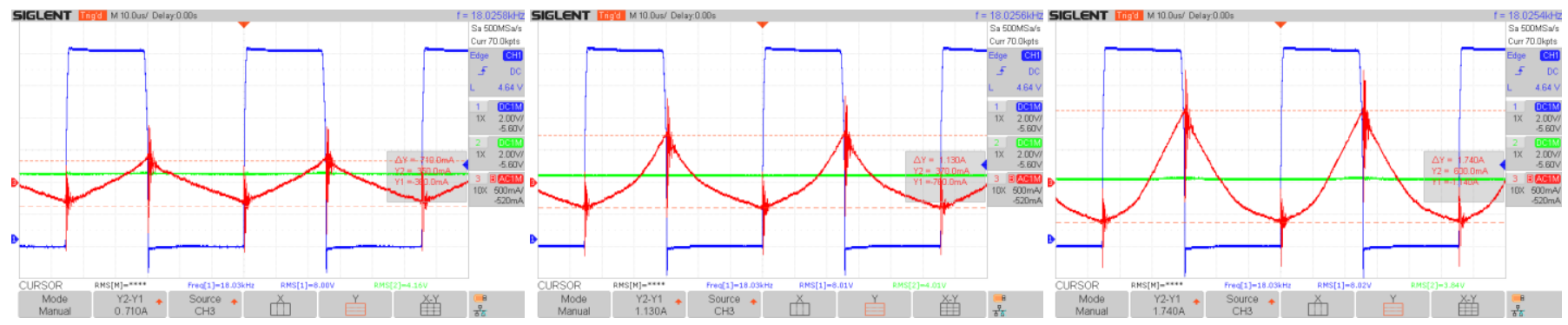

Fig. 7. Experimental measurement of the diode voltage (blue), output voltage (green) and inductor current in AC coupling (red) for different inductors and temperature conditions. First row depicts measurement conducted on the inductor $1\left(L_{n o m}=100 \mu \mathrm{H}\right)$ for $F_{s}=38 \mathrm{kHz}$ with a mean inductor current of $3.2 \mathrm{~A}$ at: a) $25^{\circ} \mathrm{C}$, b) $55^{\circ} \mathrm{C}$, c) $95^{\circ} \mathrm{C}$. Second row depicts measurement conducted on the inductor $2\left(L_{n o m}=330 \mu \mathrm{H}\right)$ for $F_{s}=18 \mathrm{kHz}$ with a mean inductor current of $1.7 \mathrm{~A}$ at: d) $25^{\circ} \mathrm{C}$, e) $55^{\circ} \mathrm{C}$, f) $95^{\circ} \mathrm{C}$.

cases we exceeded the manufacturer's limit. The switching frequency has been chosen to reach a current near the saturation value (inductance reduced to half of its maximum value), obtained by the model in correspondence of $95^{\circ} \mathrm{C}$. The oscilloscope outputs, shown in Fig. 7, contain the diode voltage (blue trace), output voltage (green trace) and the inductor current AC component (red trace). Three pictures corresponding to the environment temperature $\left(25^{\circ} \mathrm{C}\right), 55^{\circ} \mathrm{C}$, and $95{ }^{\circ} \mathrm{C}$ are given for each inductor. The corresponding measurement values are listed in Table II.

\section{A. Test on inductor 1}

As concerns the inductor 1 , the switching frequency is set to $38 \mathrm{kHz}$. Fig. 7a shows the waveforms at the environmental temperature, where it can be noted that the current exhibits a triangular waveform corresponding to a linear behavior. The maximum value of the current in this operating condition can be measured by the oscilloscope where only the AC component is shown (the reference level corresponds to the mean current, i.e., $3.2 \mathrm{~A}$ ), which is equal to $3.7 \mathrm{~A}$. During operation, the temperature rises due to the losses in the magnetic core; figures $7 \mathrm{~b}$ and $7 \mathrm{c}$ show that the current ripple in the inductor increases, as well as the maximum current. The current ripple measured at $95{ }^{\circ} \mathrm{C}$ is about $250 \%$ higher than the ripple at environmental temperature and the maximum current reaches $4.7 \mathrm{~A}$ (without considering the parasitic oscillations that can be filtered out). Moreover, a lowering of the output voltage can be appreciated; it is due to the increase of Joule drops; in particular, the output voltage from $5.76 \mathrm{~V}$ measured at $25^{\circ} \mathrm{C}$ falls to $5.55 \mathrm{~V}$ at $95{ }^{\circ} \mathrm{C}$. Since the converter is operated in an open-loop, there is no automatic increase of the duty cycle to compensate the voltage drop; however, considering that the voltage has decreased by 
"This work has been submitted to the IEEE for possible publication. Copyright may be transferred without notice, after which this version may no longer be accessible."

TABLE II

EXPERIMENTAL MEASUREMENT FOR DIFFERENT INDUCTORS AND TEMPERATURE CONDITIONS

\begin{tabular}{cccc}
\hline \hline & $\begin{array}{c}\text { Temperature } \\
\left({ }^{\circ} \mathrm{C}\right)\end{array}$ & $\begin{array}{c}\text { Output } \\
\text { voltage }(\mathrm{V})\end{array}$ & $\begin{array}{c}\text { Inductor current } \\
\text { ripple }(\mathrm{A})\end{array}$ \\
\hline Inductor 1 & 25 & 5.76 & 1.01 \\
$\left(L_{\text {nom }}=100 \mu \mathrm{H}\right)$ & 55 & 5.65 & 1.65 \\
& 95 & 5.55 & 2.54 \\
Inductor 2 & 25 & 4.16 & 0.71 \\
$\left(L_{\text {nom }}=330 \mu \mathrm{H}\right)$ & 55 & 4.01 & 1.13 \\
& 95 & 3.84 & 1.74 \\
\hline \hline
\end{tabular}

$3.6 \%$, roughly an equal rise of the duty cycle should be expected; this corresponds to increase the conduction time of the power switch from $15 \mu \mathrm{s}$ to $15.5 \mu \mathrm{s}$. In this case, the estimated peak of the current increases up to 4.9 A due to the nonlinearity.

\section{B. Test on inductor 2}

As concerns the inductor 2, the switching frequency is set to $18 \mathrm{kHz}$. Fig. 7d shows the waveforms at environmental temperature. Also in this case, it can be noted that the current shows a triangular waveform corresponding to a linear behavior. In this case, the mean current through the inductor is equal to $1.7 \mathrm{~A}$ and the maximum current is $2.1 \mathrm{~A}$. During operation, the temperature rises due to the losses in the magnetic core, and the current ripple in the inductor increases as well as the maximum current as shown in Fig. 7e and Fig. 7f. The current ripple measured at $95{ }^{\circ} \mathrm{C}$ is about $245 \%$ higher than the ripple at environmental temperature and the peak of the current reaches $2.7 \mathrm{~A}$. The output voltage decreases from 4.16 $\mathrm{V}$ to $3.84 \mathrm{~V}$ corresponding to a lowering of $7.7 \%$. To compensate it by feedback, the duty cycle has to be raised; it implies increasing the conduction time of the power switch from $25.5 \mu$ s to $27.7 \mu \mathrm{s}$. In this case, the estimated maximum value of the current reaches $2.46 \mathrm{~A}$.

In any case, the effect of the temperature starting from a linear operation leads the inductors to overtake the threshold current corresponding to saturation. Besides, the feedback further worsens the overall stress of the circuit since it tries to compensate, by increasing the duty cycle, the voltage drop. This phenomenon becomes more relevant when the power increases as the voltage drop increases as well. The overtaking of the saturation limit current is due to the FFC operation. Differently, by adopting VFC, it is possible to impose the duty-cycle $D$ by increasing the switching frequency $F_{s}$, with a $T_{o n}$ such that the current limit is not exceeded. In fact, considering that $D=T_{o n} / T=T_{o n} \cdot F_{s}$, it is possible to increase the duty cycle just by increasing the switching frequency, i.e., reducing $T_{\text {off }}$.

Figure 8 shows the curves of the temperature versus time. We stopped the tests when the temperature reached $95{ }^{\circ} \mathrm{C}$ to avoid damaging inductor windings; indeed, the insulation are made of polymers such as polyurethane, polyamide and polyester. These materials usually degrade when the temperature exceeds 80-150 ${ }^{\circ} \mathrm{C}[32]$ with consequent failure of the insulating film covering the copper and short circuits among wires. Furthermore the manufacturer guarantees a constant $\mathrm{I}_{\mathrm{RMS}}$ current up to $85^{\circ} \mathrm{C}$ [30]. Experimental data have been fitted to retrieve the exponential curve describing the temperature transient. It can

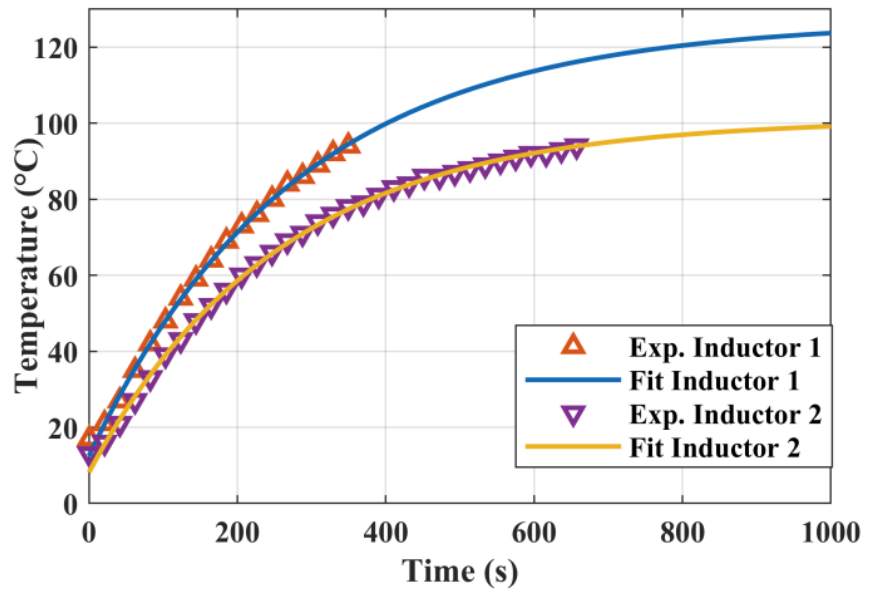

Fig. 8. Thermal transient for the inductor 1 (blue) and inductor 2 (orange).

be noted that inductor 1 reaches the maximum temperature before inductor 2, as expected since a greater current flows through it. The curve corresponding to inductor 2 tends to stabilize around the maximum temperature, whereas the curve of inductor 1 would rise to about $120^{\circ} \mathrm{C}$. It should be underlined that these transients describe the rise of the temperature starting from environmental temperature and a linear operation; the losses drive the inductor in non-linear zone, further raising the maximum peak of the current and lowering the output voltage.

The obtained results have general validity and can be applied to any inductor belonging to a DC/DC converter; they show that the temperature can drive an inductor outside the linear zone and that in the neighborhood of saturation with FFC a slight increase of the duty cycle causes a dramatic increase of the current peak.

\section{CONCLUSION}

The operating temperature of a power inductor plays a crucial role when it is operated in saturation. A full characterization of power inductors is essential to exploit the saturation region; it must encompass the magnetization curve and the temperature. The behavior of two different inductors wounded on the same core and placed in a buck converter when the saturation is exploited has been verified showing how the current profiles are varied.

The inductance characterization is based on current derivative and voltage across the inductor; some cautions ensure that the error is minimized within tolerance. Moreover, the inductor under test is characterized in real operating conditions since it is part of a switching converter, ensuring high precision measurement. The temperature of the inductor is measured at a suitable point of the core.

The measurement system gives a complete characterization of the inductance versus temperature, up to the saturation current defined as the current at which the inductance value drops at half its maximum. The measurement error is lower than the tolerance given by the manufacturer. The magnetization curve can also be retrieved, and the core saturation current versus inductance can be calculated. Experimental tests have been conceived to show how the temperature influences the current profile and, consequently, both the maximum current 
"This work has been submitted to the IEEE for possible publication. Copyright may be transferred without notice, after which this version may no longer be accessible."

through the same inductor and the output voltage of the converter. The feedback of the converter can further increase the overall stress and this is emphasized by increasing the rated power.

Finally, we demonstrated that it is possible to extrapolate the characteristics of saturation current vs. core temperature for a whole family of inductors sharing the same core once that the proportionality factor $K$ is retrieved from the measurement of one suitable inductor of the same series. This approach is thus helpful for retrieving information regarding the saturation and temperature behavior of power inductors in the non-linear region, allowing the designers to address the adverse effects of saturation.

\section{REFERENCES}

Z. Yan, C. Qimi, and Z. Junbo, "Predicting core losses under the DC bias based on the separation model," IEEE J. Emerg. Sel. Top. Power Electron., vol. 5, no. 2, pp. 833-840, 2017, doi: 10.1109/JESTPE.2017.2664882.

[2] W. Sima et al., "Low-frequency model for single-phase transformers based on the three-component Preisach model considering deep saturation," Int. J. Electr. Power Energy Syst., vol. 110, no. January, pp. 107-117, 2019, doi: 10.1016/j.ijepes.2019.02.050.

[3] M. Khalilifar, S. M. Shahrtash, M. Fateminia, and A. M. Ranjbar, "Reactor modelling with saturation characteristic and inter-phase magnetic coupling," Int. J. Electr. Power Energy Syst., vol. 123, no. March, p. 106228, 2020, doi: 10.1016/j.ijepes.2020.106228.

[4] M. S. Perdigão, J. P. F. Trovão, J. M. Alonso, and E. S. Saraiva, "Large-Signal Characterization of Power Inductors in EV Bidirectional DC-DC Converters Focused on Core Size Optimization," IEEE Trans. Ind. Electron., vol. 62, no. 5, pp. 30423051, 2015, doi: 10.1109/TIE.2015.2402632.

[5] G. Di Capua and N. Femia, "A novel method to predict the real operation of ferrite inductors with moderate saturation in switching power supply applications," IEEE Trans. Power Electron., vol. 31, no. 3, pp. 2456-2464, 2016, doi: 10.1109/TPEL.2015.2438952.

[6] G. Di Capua, N. Femia, and K. Stoyka, "Switching Power Supplies with Ferrite Inductors in Sustainable Saturation Operation," Int. J. Electr. Power Energy Syst., vol. 93, pp. 494-505, 2017, doi: 10.1016/j.ijepes.2017.06.003.

[7] V. C. Valchev and A. Van den Bossche, Inductors and Transformers for Power Electronics, 1st ed. Boca Raton, FL, USA: CRC Press, 2018.

[8] M. W. Beraki, J. P. F. Trovao, M. S. Perdigao, and M. R. Dubois, "Variable Inductor Based Bidirectional DC-DC Converter for Electric Vehicles," IEEE Trans. Veh. Technol., vol. 66, no. 10, pp. 8764-8772, 2017, doi: 10.1109/TVT.2017.2710262.

[9] G. Vitale, G. Lullo, and D. Scirè, "Thermal stability of a DC/DC converter with inductor in partial saturation," IEEE Trans. Ind. Electron., 2020, doi: 10.1109/TIE.2020.3014580.

[10] F. Bizzarri, M. Lodi, A. Oliveri, A. Brambilla, and M. Storace, "A nonlinear inductance model able to reproduce thermal transient in SMPS simulations," in Proceedings - IEEE International Symposium on Circuits and Systems, May 2019, vol. 2019-May, pp. 1-5, doi: 10.1109/ISCAS.2019.8702418.

[11] A. Oliveri, G. Di Capua, K. Stoyka, M. Lodi, M. Storace, and N. Femia, "A power-loss-dependent inductance model for ferrite-core power inductors in switch-mode power supplies," IEEE Trans. Circuits Syst. I Regul. Pap., vol. 66, no. 6, pp. 2394-2402, Jun. 2019, doi: 10.1109/TCSI.2018.2889856.

[12] A. Oliveri, M. Lodi, and M. Storace, "Accurate Modeling of Inductors Working in Nonlinear Region in Switch-Mode Power Supplies with Different Load Currents," in SMACD 2018 - 15th International Conference on Synthesis, Modeling, Analysis and Simulation Methods and Applications to Circuit Design, 2018, pp. 233-236, doi: 10.1109/SMACD.2018.8434871.

[13] P. Burrascano, G. Di Capua, S. Laureti, and M. Ricci, "Neural models of ferrite inductors non-linear behavior," in Proceedings - IEEE International Symposium on Circuits and Systems, 2019, vol. 2019May, doi: 10.1109/ISCAS.2019.8702466.
[14] D. Scire, G. Lullo, and G. Vitale, "Design and Modeling of an Interleaving Boost Converter with Quasi-Saturated Inductors for Electric Vehicles," in 2020 AEIT International Conference of Electrical and Electronic Technologies for Automotive (AEIT AUTOMOTIVE), Nov. 2020, pp. 1-6, doi: 10.23919/AEITAUTOMOTIVE50086.2020.9307424.

[15] G. Migoni, M. E. Romero, F. Bergero, and E. Kofman, "A Mixed Modeling Approach for Efficient Simulation of PWM Switching Mode Power Supplies," IEEE Trans. Power Electron., vol. 34, no. 10, pp. 9758-9767, Oct. 2019, doi: 10.1109/TPEL.2019.2891174.

[16] K. Stoyka, N. Femia, and G. Di Capua, "Power Inductors Behavioral Modeling Revisited," IEEE Trans. Circuits Syst. I Regul. Pap., vol. 67, no. 12, pp. 5636-5649, Dec. 2020, doi: 10.1109/TCSI.2020.3002615.

[17] N. Femia, G. Di Capua, and K. Stoyka, "Saturation and thermal stability of power inductors," in ICECS 2020 - 27th IEEE International Conference on Electronics, Circuits and Systems, Proceedings, Nov. 2020, pp. 1-4, doi: 10.1109/ICECS49266.2020.9294851.

[18] M. Uno, "A Direct-Reading Instrument for Measurements of Inductance of Ferrite-Core Coils," IEEE Trans. Instrum. Meas., vol. IM-31, no. 2, pp. 139-140, Jun. 1982, doi: 10.1109/TIM.1982.6312540.

[19] B. Waltrip and F. Seifert, "A programmable capacitor for inductance measurements," IEEE Trans. Instrum. Meas., vol. 66, no. 6, pp. 1572-1578, Jun. 2017, doi: 10.1109/TIM.2017.2659818.

[20] J. Lu, D. A. Pan, B. Yang, and L. Qiao, "Wideband magnetoelectric measurement system with the application of a virtual multi-channel lock-in amplifier," Meas. Sci. Technol., vol. 19, no. 4, 2008, doi: 10.1088/0957-0233/19/4/045702.

[21] C. Fernandez, Z. Pavlovic, S. Kulkarni, P. McCloskey, and C. O'Mathuna, "Novel High-Frequency Electrical Characterization Technique for Magnetic Passive Devices," IEEE J. Emerg. Sel. Top. Power Electron., vol. 6, no. 2, pp. 621-628, 2018, doi: 10.1109/JESTPE.2018.2798919.

[22] A. J. Batista, J. C. S. Fagundes, and P. Viarouge, "An Automated Measurement System for Core Loss Characterization," IEEE Trans. Instrum. Meas., vol. 48, no. 2, pp. 663-667, Apr. 1999, doi: $10.1109 / 19.769682$.

[23] T. Cheng, D. D. C. Lu, and Y. Siwakoti, "Electro-Thermal Modeling of a Boost Converter Considering Device Self-heating," 2019 IEEE 4th Int. Futur. Energy Electron. Conf. IFEEC 2019, 2019, doi: 10.1109/IFEEC47410.2019.9014948.

[24] K. Górecki and K. Detka, "Application of Average Electrothermal Models in the SPICE-Aided Analysis of Boost Converters," IEEE Trans. Ind. Electron., vol. 66, no. 4, pp. 2746-2755, 2019, doi: 10.1109/TIE.2018.2847694.

[25] M. Thone and J. Wens, "MADMIX The Standard for Measuring SMPS Inductors," Bodo's Power Systems, pp. 52-54, 2015.

[26] F. C. Lee, S. Wang, and Q. Li, "Next Generation of Power SuppliesDesign for Manufacturability," IEEE J. Emerg. Sel. Top. Power Electron., vol. 6777, no. c, pp. 1-1, Jun. 2020, doi: 10.1109/jestpe.2020.3002857.

[27] H. Matsumori, T. Shimizu, X. Wang, and F. Blaabjerg, "A practical core loss model for filter inductors of power electronic converters," IEEE J. Emerg. Sel. Top. Power Electron., vol. 6, no. 1, pp. 29-39, 2018, doi: 10.1109/JESTPE.2017.2761127.

[28] G. Lullo, S. Rosato, D. Scirè, and G. Vitale, "Characterization of Non-linear Inductors Including Thermal Effects for Power Applications," Renew. Energy Power Qual. J., vol. 1, no. April, pp. 728-734, Apr. 2018, doi: 10.24084/repqj16.446.

[29] C. W. T. McLyman, Transformer and Inductor Design Handbook, 4th ed. Boca Raton, FL, USA: CRC Press, 2017.

[30] Coilcraft Inc., "SMT Power Inductors - DO5010H Series." https://www.coilcraft.com/en-us/products/power/unshieldedinductors/ferrite-drum-surface-mount/do/do5010h/ (accessed Sep. 01, 2020).

[31] R. W. Erickson and D. Maksimović, Fundamentals of Power Electronics. Boston, MA: Springer US, 2001.

[32] A. Barili, A. Brambilla, G. Cottafava, and E. Dallago, "A Simulation Model for the Saturable Reactor," IEEE Trans. Ind. Electron., vol. 35, no. 2, pp. 301-306, 1988, doi: 10.1109/41.192663. 